\title{
Editorial
}

\section{End-to-End Automation of 5G Networks}

\author{
Tara Ali Yahiya $\mathbb{D}^{1},{ }^{1}$ Pinar Kirci $\mathbb{D D}^{2}{ }^{2}$ and André-Luc Beylot ${ }^{3}$ \\ ${ }^{1}$ Department of Computer Science and Engineering, University of Kurdistan-Hewler, Erbil, KRG, Iraq \\ ${ }^{2}$ Department of Engineering Sciences, Faculty of Engineering, Istanbul University-Cerrahpasa, Istanbul, Turkey \\ ${ }^{3}$ Department of Telecommunications and Networks, ENSEEIHT, University of Toulouse, Toulouse, France
}

Correspondence should be addressed to Tara Ali Yahiya; t.ibrahim1@ukh.edu.krd

Received 28 April 2019; Accepted 28 April 2019; Published 17 June 2019

Copyright ( $\odot 2019$ Tara Ali Yahiya et al. This is an open access article distributed under the Creative Commons Attribution License, which permits unrestricted use, distribution, and reproduction in any medium, provided the original work is properly cited.

The fifth-generation (5G) network will be the next big revolution in the area of mobile and wireless communication. This is not due only to the high data rate that it is offering but also due to its openness to different types of applications like the ultralow latency applications, Internet of things (IoT), and massive device-to-device communications. These kinds of applications cannot be supported without the big changes witnessed by the core network of the $5 \mathrm{G}$ through the virtualization and softwarization techniques that revolutionize not only the core but also the radio network, bringing higher performance, low latency, and quality of service guarantee to these types of applications.

The revolution in $5 \mathrm{G}$ networks is represented by their automation and adaptability to fulfill different requirements of users. To do so, the service and the network infrastructure are configured through techniques like software-defined networking (SDN) and network function virtualization (NFV) to enable the dynamicity of both radio and core networks instead of having a fixed and static architecture. However, this automation cannot be achieved only through the use of tools but can also be achieved by using intelligent mechanisms and predictive analytics in order to make them responsive to different customers' requirements.

Providing techniques of automation in both radio and core networks will provide reliability through collaborative intelligent resource allocation management. Thus, this will result in providing agile services and new on-demand services in order to meet the requirements of the users as well as their quality of service (QoS), especially for realtime services.
The article "Mobile Edge Assisted Live Streaming System for Omnidirectional Video" by X. Hu et al. proposes a mobile edge assisted live streaming system for omnidirectional video (MELiveOV); the MELiveOV can intelligently offload the processing tasks to the edge computing-enabled $5 \mathrm{G}$ base stations. The MELiveOV consists of an omnidirectional video generation module, a streaming module, and a viewpoint prediction module. A prototype system of MELiveOV is implemented to prove its complete end-to-end OV live streaming service. The evaluation result demonstrates that compared with the traditional solution, the MELiveOV can reduce the network bandwidth requirement by about $50 \%$ and the transmission delay by more than $70 \%$ while ensuring the quality of the user's experience.

The article "An End-to-End Automation Framework for Mobile Network Testbeds" by A. D. Zayas et al. describes the end-to-end automation framework developed as part of the TRIANGLE project. The TRIANGLE project is devoted to the benchmarking of apps and devices in mobile networks. For that purpose, it is needed to ensure the repeatability in the behaviour of all the components of the mobile network during the execution of the same test. This is why one of the main objectives of the project was to develop an end-to-end automation framework to provide repeatable testing. The paper describes in detail the design and the implementation of the framework.

The article "NSAF: An Approach for Ensuring ApplicationAware Routing Based on Network QoS of Applications in SDN" by J. Park et al. proposes the network situation-aware framework (NSAF) to more efficiently handle application routing based on the QoS requirements and changing network status. A 
mechanism for supporting the NSAF consisting of application registration, network status monitoring, violation detection, and routing control is also presented. The applicability and feasibility of the proposed NSAF are verified by implementing a prototype. The NSAF may be used as a reference model for efficiently managing SDN-based networks to ensure application QoS.

The article "Expanding GÉANT Testbeds Service to Support Pan-European 5G Network Slices for Research in the EuWireless Project" by Á. Rios et al. presents the design options for creating a Pan-European mobile network for research in the context of the European Horizon 2020 EuWireless project. The most likely direction is a platform that makes it easier to create network slices for research. In this context, we identify one promising technology to implement network slicing in 5G networks: the framework GÉANT Testbeds Service (GTS). GTS is currently a production service by GÉANT that offers remote construction and use of virtual testbeds for wired networks mapped to the real GÉANT infrastructure. These GTS virtualized testbed environments conform to software-defined network (SDN) principles and offer computing, storing, and switching resources, at scale and with line rate performance. In this paper, we explain how the current (wire oriented) GTS can be extended with the $5 \mathrm{G}$ components, such as radio access nodes (gNBs), transport networks, and user devices, in order to implement $5 \mathrm{G}$ network slices. Our first conclusion is that using GTS for EuWireless implementation is feasible, dramatically increasing the potential impact of this service in the research community.

In the article "Independence and Fairness Analysis of $5 \mathrm{GmmWave}$ Operators Utilizing Spectrum Sharing Approach" by M. L. Attiah et al., an analytical framework involving a flexible hybrid mmWave SSA is presented to assess the effectiveness of SSA and investigate its influence on network functionality in terms of independence and fairness among operators. Two mmWave frequencies $(28 \mathrm{GHz}$ and $73 \mathrm{GHz})$ are used with different spectrum bandwidths. Various access models have been presented for adoption by four independent mobile network operators that incorporate three types of spectrum allocation (exclusive, semipooled, and fully pooled access). Furthermore, an adaptive multistate mmWave cell selection scheme is proposed to associate typical users with the tagged mmWave base stations that provide a great signal-to-interferenceplus-noise ratio, thereby maintaining reliable connections and enriching user experience. Numerical results show that the proposed strategy achieves considerable improvement in terms of fairness and independence among operators, which paves the way for further research activities that would provide better insight and encourage mobile network operators to rely on SSA.

\section{Conflicts of Interest}

The editors declare that there are no conflicts of interest regarding the publication of this special issue.

\section{Acknowledgments}

The editors would like to thank the authors for their contributions and the reviewers of the papers for their help in bringing this issue to its current form.

Tara Ali Yahiya Pinar Kirci André-Luc Beylot 


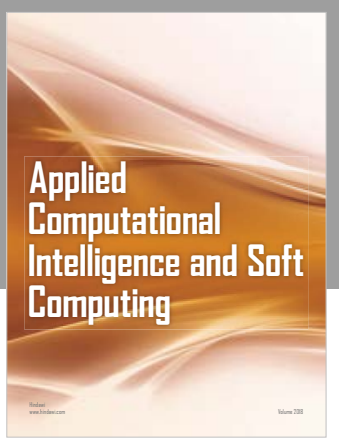

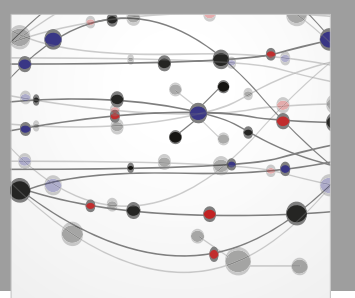

The Scientific World Journal
Submit your manuscripts at

Computing
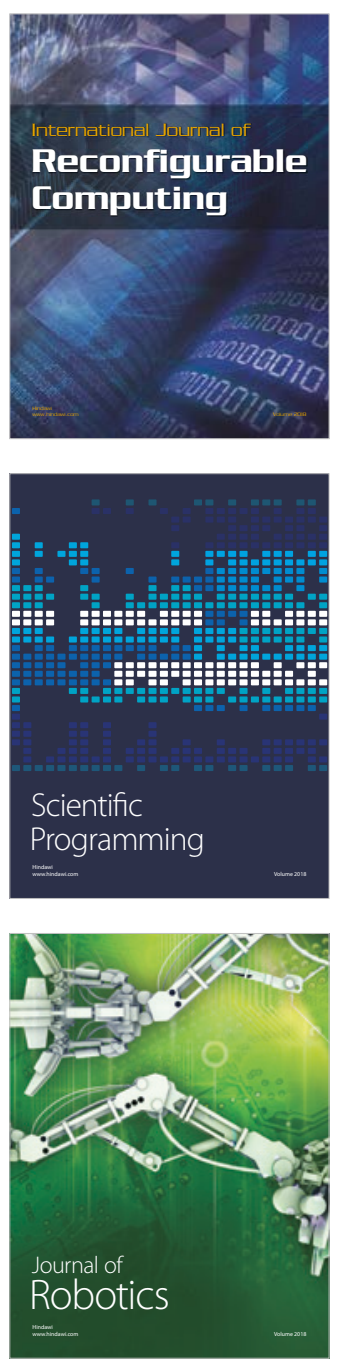

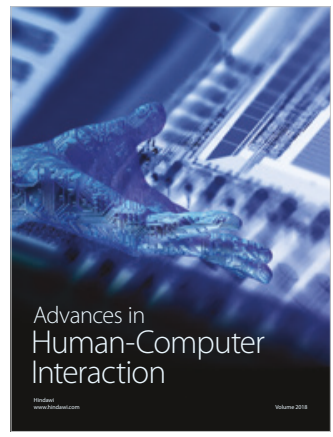

Human-Compute

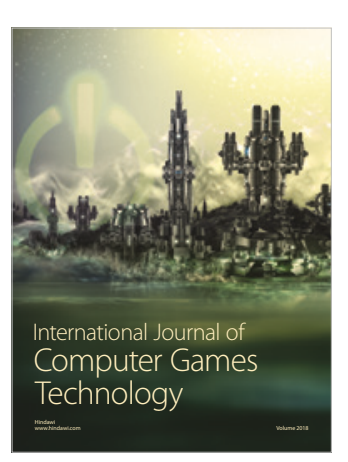

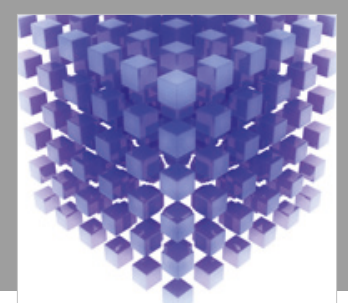

Mathematical Problems in Engineering

\section{Engincering}
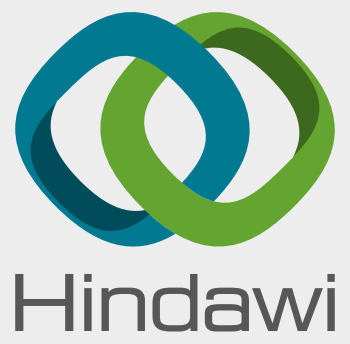

www.hindawi.com
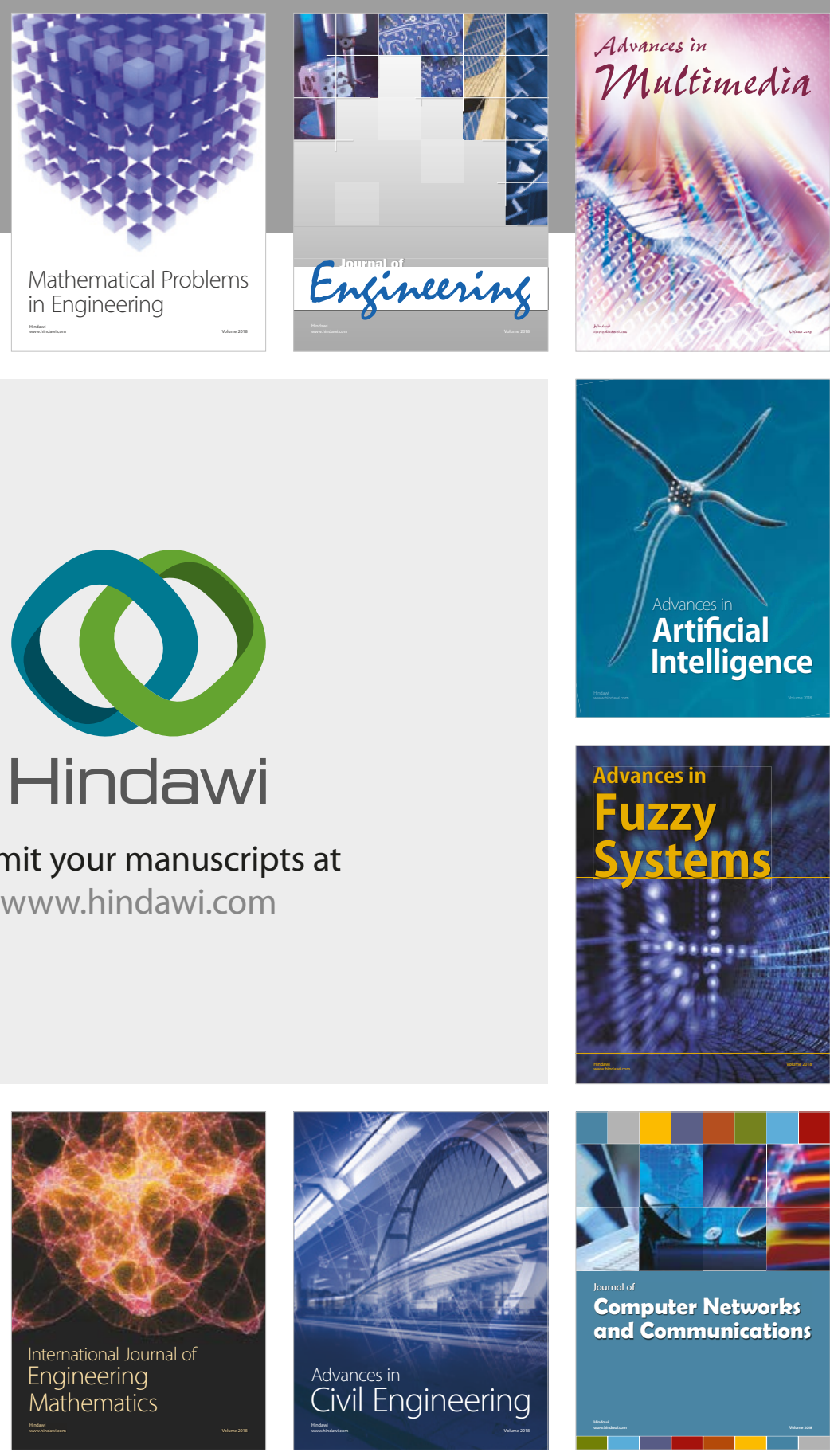

Computer Networks and Communications

Multimedia
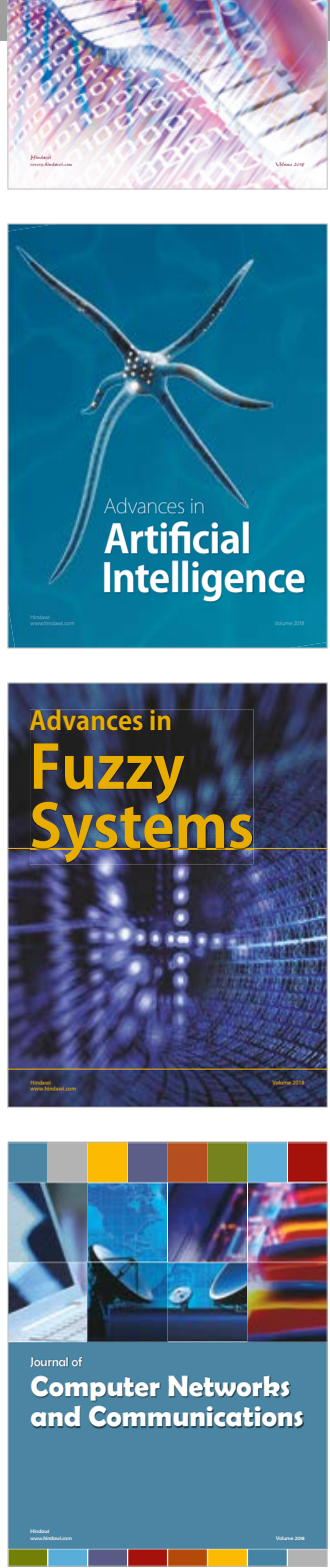

Advances in

Modelling \&

Simulation

in Engineering

interaction

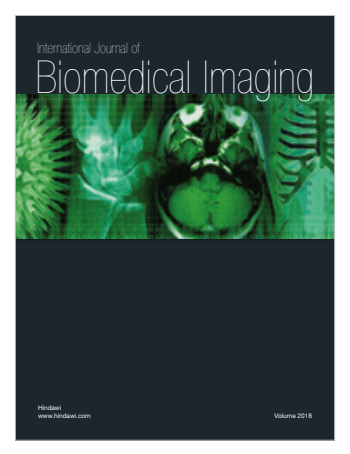

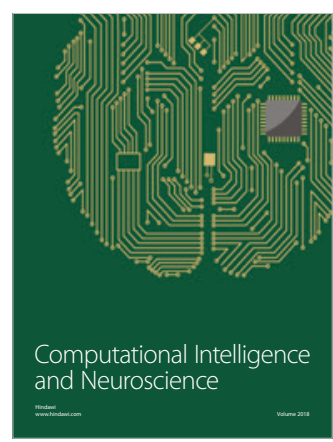

